\title{
Combined effects of planning and execution constraints on bimanual task performance
}

\author{
Loes Janssen • Marieke Beuting • Ruud Meulenbroek • \\ Bert Steenbergen
}

Received: 7 April 2008 / Accepted: 15 August 2008 / Published online: 28 August 2008

(C) The Author(s) 2008. This article is published with open access at Springerlink.com

\begin{abstract}
In this study we investigated the relative impact of planning and execution constraints on discrete bimanual task performance. In particular, in a bimanual CD-placement task, we compared people's preference to end movements comfortably with their preference to move symmetrically. In "Experiment 1" we examined the degree of interlimb coupling as participants repositioned two CDs in a CD rack by simultaneously moving their arms mirrorsymmetrically or asymmetrically into comfortable or uncomfortable end postures. Interlimb coupling was stronger when the arms moved symmetrically towards uncomfortable end postures. In "Experiment 2" participants were asked to realize specific end orientations of the CDs but they were free to choose an initial grip type and subsequent direction of forearm rotation. Surprisingly, the participants did not move their arms symmetrically but preferred to end in a comfortable posture with their right hand but not with their left hand. We conclude that in discrete bimanual task performance the tendency to end movements in a comfortable posture dominates over the tendency to synchronously activate homologous muscle pairs. The lateralized end-state comfort effect suggests a hemispheric specialization for motor planning.
\end{abstract}

Keywords Bimanual coordination - Motor planning · Movement symmetry $\cdot$ Object manipulation . Hemispheric lateralization

L. Janssen $(\square) \cdot$ M. Beuting $\cdot$ R. Meulenbroek · B. Steenbergen Nijmegen Institute for Cognition and Information,

Radboud University Nijmegen, PO Box 9104,

6500 HE Nijmegen, The Netherlands

e-mail: 1.janssen@nici.ru.nl

\section{Introduction}

Every day we grasp and manipulate objects, often seemingly even without thinking or spending any effort. However, to do so optimally, we need to plan our movements before we undertake any action (Rosenbaum et al. 2001). For example, when you want to eat with knife and fork, you pick up the cutlery in such a way that they can immediately be used for eating, even if the knife lies upside down. Thus, the forthcoming action, viz., eating, is anticipated in the grip type, i.e. the orientation of the hand that is used when picking up the cutlery. Anticipation of a forthcoming action is demonstrated to have effects on reaching kinematics (Marteniuk et al. 1987; Johnson-Frey et al. 2004) and joint couplings during reaching (Steenbergen et al. 1995). In the example of eating with knife and fork, anticipatory planning is evidenced by the macroscopic variable grip type. The knife is picked up with a full grip with the thumb pointing towards the blade of the knife. However, if you pick up a knife in order to pass it to someone else, you probably adopt another grip type, with the thumb pointing towards the handle or at least such that the other person can take the knife without any danger. Rosenbaum et al. (1992) studied this phenomenon of grip type anticipation and had participants reach for a bar that had to be moved as quickly as possible from a home location to a target location. Participants generally took hold of the bar in a way that afforded a comfortable posture at the target location even when this necessitated an uncomfortable posture at the home location (Rosenbaum et al. 1992). This phenomenon was termed the end-state comfort effect. Here, comfort was defined as the degree to which the joints of the multijoint effector system are in the middle of their range of motion (Rossetti et al. 1994). The end-state comfort effect can also be deduced from the cognitive theory of posture-based motion planning 
by Rosenbaum et al. (2001), which presumes that planning of the goal posture precedes the execution of the movement.

Thus far, the end-state comfort effect has mainly been studied for discrete, unimanual tasks. However, more recently researchers have also focused their attention on the end-state comfort effect in bimanual tasks (Fischman et al. 2003; Weigelt et al. 2006; Hughes and Franz 2008). As an example, Fischman et al. (2003) showed that participants adjusted the height of their start grips whenever they had to place a dowel at a very low target location (prompting a high start grip) or at a very high target location (eliciting a low start grip). In line with this, Weigelt et al. (2006) showed in a bimanual object manipulation task a strong tendency for both hands to end comfortably. Their results suggest that planning for comfortable end-states dominates over processes related to movement execution of both hands. In contrast to Weigelt et al. (2006), a large number of experimental studies, predominantly examining rhythmic cyclical tasks, did show the presence of interlimb interference at the level of movement execution. These interference effects were especially obvious when nonhomologous muscle pairs were activated ( $\mathrm{Li}$ et al. 2004). Ample studies showed an increased stability of arm movements in the symmetric mode (both hands/arms moving mirror-symmetrically with respect to the longitudinal axis of the body) compared to arm movements in the asymmetric mode (both hands/arms moving alternating with respect to the longitudinal axis of the body) (Byblow et al. 1994; Semjen et al. 1995; Swinnen et al. 1997, 1998; Li et al. 2004).

According to the parameter-specification model of Heuer (1993), intermanual interference arises whenever different parameter values have to be specified independently for the two hands. Indeed, when participants had to move a manipulandum to different amplitudes, interference effects arose, i.e. the amplitude and movement time of the one hand scaled with the amplitude and movement time of the other hand. (Spijkers and Heuer 1995; Spijkers et al. 1997; see Bingham et al. 2008 for theses effects in bimanual object manipulation tasks). However, recent research findings challenged this model as interference effects at the execution level appeared to be subsidiary to the effects related to planning. Kunde and Weigelt (2005) showed a lack of the effect of moving in symmetry in an object-manipulation task. Instead, the congruency of the intended object orientations proved to have a larger effect on movement performance. Motorsymmetry effects only became substantial when the movements themselves became the action goal (Kunde and Weigelt 2005). Similarly, Diedrichsen et al. (2001) showed that bimanual interference is reduced or even disappears when direct cues were used instead of centrally spaced symbolic cues (e.g., letters, colors) in a pointing task. They suggested that the cost to initiate asymmetric movements does not arise from spatial interference in a central motor-programming stage, but is rather a consequence of interference in the translation of symbolic information into motor commands (see also Weigelt et al. 2007).

These findings beg the question as to whether the tendency to move symmetrically is strong enough to affect goal-related action planning and the forthcoming movement execution in a discrete bimanual task. Stated differently, does either the tendency to move in symmetry or the goal-related action planning dominate task performance in a discrete bimanual object manipulation task? Although participants in the study of Weigelt et al. (2006) showed goal-related action planning (because they showed the endstate comfort effect) for both hands, the design of that study did not include 'critical conditions', in which a choice for one constraint is at the expense of the other. Stated differently, the constraint with respect to movement symmetry was not very strict. In addition, they only included the macroscopic variable grip type. In the present study, we systematically investigated the impact of both types of constraints via examination of bimanual-interference effects related to end-state planning and movement execution. To scrutinize the effects of movement symmetry and end-state comfort at a more microscopic level, we included kinematic analyses of the bimanual movements that we studied.

We conducted two experiments. In "Experiment 1" we focused on the effects of symmetry of moving and comfort of the end posture on interlimb coordination. The end posture of each hand was explicitly instructed. In this way, we could control the conditions and isolate the effect of end comfort on the kinematics of the bimanual movements. In "Experiment 2" participants were free to choose handgrips and movement trajectories. Only the end orientation was cued. In this way, we assessed whether participants would choose to end comfortably in favor of moving symmetrically and if so, whether there would be differences with respect to the preferred and non-preferred hand in prioritizing the planning constraint.

If planning principles associated with goal states prevail, this would be a strong indication that the performance of discrete bimanual movements is related to end goals rather than to the movements required to reach these goals. Such a finding would challenge the parameter-specification model of Heuer (1993). Conversely, if a shift in prioritization towards symmetrical movements occurs, this would point to a need to reformulate existing motion-planning theories that claim that goals are always prioritized, such as is the case in the posture-based motion-planning theory (Rosenbaum et al. 2001). 


\section{Experiment 1}

To investigate the relative impact of the planning (end comfort) and the execution (movement symmetry) constraints in a bimanual object-manipulation task, we designed a bimanual CD-placement task.

Methods (1)

\section{Participants}

Ten female university students (mean age $=20.7$ years/ months, $\mathrm{SD}=1.9$ years/months) participated in the experiment. All participants were right-handed as confirmed by a score of $\geq 80$ on the ten-item version of the Edinburgh Handedness Inventory (Oldfield 1971). Informed consent from all participants was obtained prior to the experiment. Participants were naive with regard to the purpose of the study and received course credits for their participation. The experiments were conducted conform the standards of the declaration of Helsinki and in accordance with local ethical guidelines.

\section{Experimental setup}

Figure 1 shows the general layout of the experimental setup. The setup consisted of two square $C D$ boxes $(15 \mathrm{~cm} \times 15 \mathrm{~cm})$, in which a CD casing could be placed in either a horizontal or vertical orientation. Each box was supplied with two green light-emitting diodes (LEDs) that served as visual cues. The LED on the top of the box indicated a vertical placement of the $\mathrm{CD}$, whereas the LED on the side indicated a horizontal placement. Participants were comfortably seated right in front of the experimental setup, which was placed on a table with the boxes at participants'

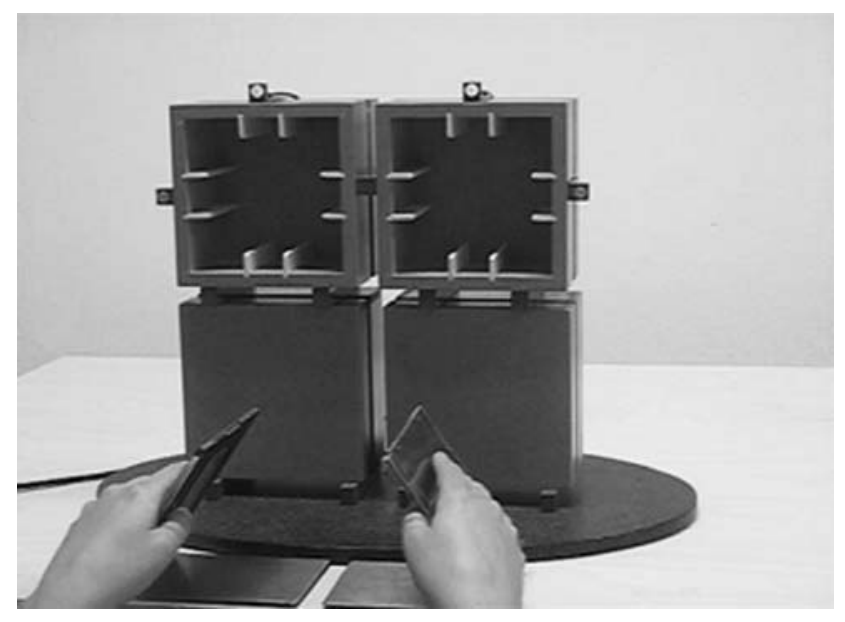

Fig. 1 Photograph of the experimental setup from the viewpoint of a participant eye height. At the start of each trial, one or two CD casings (for unimanual or bimanual tasks, respectively) were present at two CD holders, which we located on the table $7 \mathrm{~cm}$ in front of the $\mathrm{CD}$ boxes. Both $\mathrm{CD}$ holders could be placed in two orientations: $45^{\circ}$ or $-45^{\circ}$ with regard to the vertical, viz, upright orientation.

\section{Experimental design and procedure}

At the start of each trial participants held one (in unimanual conditions) or two (in bimanual conditions) CD casing(s) that rested in the holder(s), with the left hand, the right hand, or both hands. The trial started with a single tone and after a random inter-stimulus interval of $0.5-2.5 \mathrm{~s}$, one (in unimanual conditions) or two (in bimanual conditions) of the LEDs were switched on providing the visual cue for CD placement. We instructed participants to react to the visual cue as fast and accurate as possible by placing the CD casing(s) in the CD box(es) in the cued orientation. Participants were instructed to keep their hand(s) in the end position for several seconds until the LED(s) switched off. Subsequently, the CD casing(s) could be replaced in its initial starting position.

All participants performed both unimanual and bimanual tasks. In all trials, the $\mathrm{CD}(\mathrm{s})$ had an oblique start orientation, either $-45^{\circ}$ or $+45^{\circ}$ with respect to the vertical. As such, the start grips had the following postures; $45^{\circ}$ supination of the forearm, $45^{\circ}$ pronation of the forearm, or $135^{\circ}$ pronation of the forearm (Fig. 2a). The end postures were either horizontal or vertical and were reached by either a pronation or supination of the forearm over a range of $45^{\circ}$ (Fig. 2b). Thus, we made sure that in all conditions, the same degree of forearm rotation was needed to perform the task. Manipulation of the start and end orientations resulted in 12 unique unimanual conditions ( 3 start orientations $\times 2$ end orientations $\times 2$ hands). In the bimanual conditions, orientation of the start posture could be parallel (one hand $45^{\circ}$ forearm pronation and the other $45^{\circ}$ forearm supination or $135^{\circ}$ pronation, or vice versa) or non-parallel (both fore$\operatorname{arms~} 45^{\circ}$ supination or $45^{\circ}$ pronation). Parallel start orientations induced asymmetric movements, whereas the nonparallel start orientations induced symmetric movements with respect to the longitudinal axis of the body. The end orientations in the bimanual trials were always congruent, i.e., for both hands either horizontal or vertical. This yielded 12 bimanual conditions [ 6 start grips ( 4 parallel +2 non-parallel) $\times 2$ end grips].

Participants performed a grand total of 240 trials, subdivided in 12 blocks of 10 unimanual trials and 12 blocks of 10 bimanual trials. Each block consisted of two conditions ( 5 trials of each condition) with the same start grip, but different end orientations. The order of these blocks was randomized with the restriction that unimanual and 
Fig. 2 Start postures (a) and end postures (b) of the right hand investigated in Experiment 1

a) Start postures

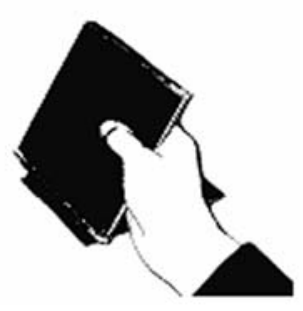

$45^{\circ}$ supination

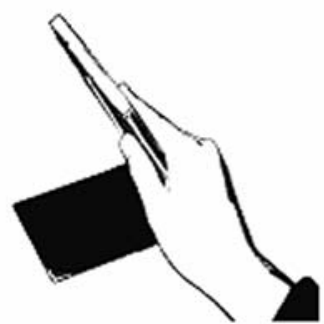

$45^{\circ}$ pronation

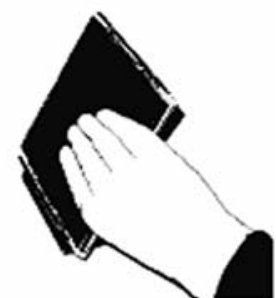

$135^{\circ}$ pronation

b)

End postures

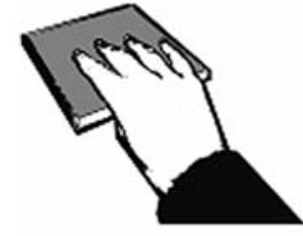

horizontal overhand

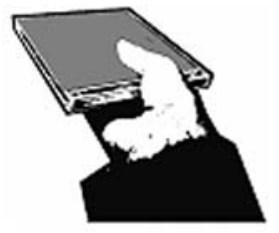

horizontal undemand

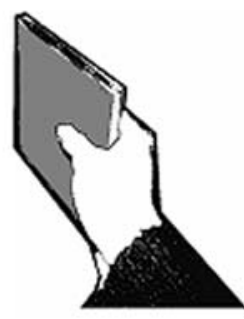

vertical thumb up

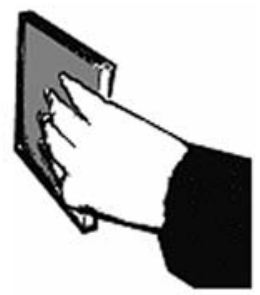

vertical thumb down bimanual blocks were alternated. Furthermore, the order of the trials was randomized within the blocks. If the participant ended the movement in the wrong orientation, or when the recording of the wrist movement failed, the trial was repeated at the end of that block.

Prior to the start of the experiment, participants performed a few practice trials to check whether the task was understood correctly, and to familiarize themselves with the task. The total duration of the experiment was about $1 \mathrm{~h}$.

\section{Data acquisition}

Hand movements were registered by two OPTOTRAK systems (Optotrak 3020, Nothern Digital Inc.) with a sample rate of $100 \mathrm{~Hz}$. Both cameras were placed at approximately $90^{\circ}$ of each other at a height of approximately $2 \mathrm{~m}$ to enhance visibility of the Infrared Emitting Diodes (IREDs). On each wrist of the participant we attached a rigid body that consisted of a wrist band with a metal plate on the dorsal side of the wrist on which four IREDs were fixed in a rectangular fashion. We checked the position of the rigid bodies around the wrists during the practice trials to ensure that at least three of the IREDs were in view of one of the two Optotrak cameras during task performance. The coordinate frame of the Optotrak system was oriented such that $x, y$ and $z$-axis corresponded to the horizontal, posterioranterior and vertical dimension with respect to the participant. Recordings started as the LED(s) turned on and ended after completion of the task. The whole experiment was videotaped using a digital camera, for future reference.

Next to movement registration we collected comfort ratings of the different postures that could be adopted at the start and the end of the trial. For this purpose, we asked participants to adopt a start or an end posture and to give a rating between 1 and 5 reflecting comfort of the posture, with 1 being very uncomfortable and 5 being very comfortable. Next, the participant was asked to change the grip type and to score this newly adopted posture. This procedure was repeated until the comfort scores of three different start postures $\left(45^{\circ}\right.$ supinated, $45^{\circ}$ pronated and $135^{\circ}$ pronated) and four different end postures $\left[0^{\circ}\right.$ (vertical with thumb up), $-90^{\circ}$ (horizontal supination), $+90^{\circ}$ (horizontal pronation) and $+180^{\circ}$ (vertical with thumb down)] were assessed for each hand (Fig. 2). Comfort was assessed twice, once before and once after the experiment.

\section{Data analysis}

The raw Optotrak signals were partially interpolated with cubic spline interpolation (up to ten successive samples, 
corresponding to $100 \mathrm{~ms}$ ), which never occurred before movement initiation. Thereafter, they were low-pass filtered using a second order Butterworth filter (cut-off frequency of $10 \mathrm{~Hz}$ ). The three-dimensional position data were differentiated to velocity values in three dimensions, which were integrated to obtain speed values. We defined reaction time (RT) as the point at which speed signals exceeded $5 \%$ of the maximum speed in that trial. Movement time (MT) was defined for the object transport phase, i.e. the time between RT and the first local minimum after maximum speed was reached. In addition, we calculated the relative duration in \% MT of the acceleration and deceleration phases. Movement onsets, maximum speeds and movement offsets were generated semi-automatically using custom-made selection routines and checked visually. RTs and MTs that deviated more than three standard deviations from the mean in that particular condition were discarded from further analyses. This occurred in 3.5\% of the trials.

Interlimb coupling was determined by comparing rotations of both wrists along the forearm axis. We calculated Pearson's correlation coefficient between the rotation signals of both wrists as a measure of interlimb coupling strength. The correlations were calculated in the interval from the start of the trial, i.e., $\operatorname{LED}(\mathrm{s})$ switched on, until the end of the movement time. In addition, this interval was subdivided in two parts: the acceleration time (before peak speed) and the deceleration time (after peak speed). Correlations between the wrist rotations were also calculated for these two intervals separately.

Depending on the research question and type of dependent variable under investigation, we applied repeated-measures ANOVAs and paired $T$ tests, which will be described separately in the relevant paragraphs of the results section.

\section{Results (1)}

\section{Comfort ratings}

Participants gave ratings to three different start postures and four different end postures per hand. As the ratings given before the experiment did not differ from those given after the experiment, we report the mean ratings of both measurements. The ratings were evaluated using two repeated measurements ANOVAs, one for the start postures and one for the end postures. The designs consisted of two withinsubject factors, Hand (two levels: left or right) and Posture (three levels for the start postures: $45^{\circ}$ supination, $45^{\circ}$ pronation, and $135^{\circ}$ pronation, and four levels for the end postures: horizontal overhand, horizontal underhand, vertical thumb up, and vertical thumb down).

For the start postures a main effect of Posture was observed $[F(2,18)=70.49, P<0.01]$. Post hoc pair wise comparisons revealed that only comfort of the $135^{\circ}$ prona- tion posture (mean comfort $=1.88$ ) differed significantly from the other two postures [mean comfort $45^{\circ}$ pronation $=4.78, t(9)=19.96, P<0.01 ;$ mean comfort $45^{\circ}$ supination $=3.93, t(9)=7.43, P<0.01]$.

For the end postures we observed main effects of both Hand $\quad[F(1,9)=7.97, \quad P<0.05] \quad$ and Posture $[F(3,27)=35.70, P<0.01]$. Overall ratings for the left hand (mean comfort $=3.21$ ) were slightly lower than those for the right hand (mean comfort $=3.53$ ). Furthermore, post hoc analysis revealed that participants gave higher ratings (indicating more comfort) to the horizontal overhand posture than to the horizontal underhand posture $[t(9)=2.83$, $P<0.05]$. For the vertical end postures, the posture with the thumb up was rated more comfortable than the posture with the thumb down $[t(9)=16.14, P<0.01]$. Therefore, we defined the horizontal overhand posture and the vertical posture with the thumb up as comfortable end postures, and the horizontal underhand posture and the vertical posture with the thumb down as uncomfortable end postures.

\section{Symmetry effects}

In order to study the effects of movement symmetry, we performed two repeated measures ANOVAs, one for RT and one for MT. Therefore, we selected conditions in which only the factor of interest (i.e. symmetry) was manipulated while other factors (i.e. start and end posture comfort) were kept constant. Accordingly, we included four conditions, all with vertical, comfortable, end postures (i.e. with the thumb up). In order to avoid interference with start and end posture comfort, horizontal ending conditions were excluded from this analysis, as they required either the $135^{\circ}$ pronation start posture or an underhand end posture, which are both less comfortable. Two conditions had parallel start orientations (one hand $45^{\circ}$ supination and the other hand $45^{\circ}$ pronation) inducing asymmetric movements, and the other two conditions had non-parallel start postures (both hands $45^{\circ}$ pronation or both hands $45^{\circ}$ supination) inducing symmetric movements (Fig. 3). In addition, we used four unimanual control conditions in which the hands had the same start postures $\left(45^{\circ}\right.$ pronation and $45^{\circ}$ supination) and also ended vertically with the thumb up, thus similar to the postures of both hands in the bimanual conditions. The ANOVA designs consisted of one within-subject factor Symmetry (three levels: symmetric, asymmetric or unimanual). For both the RT and MT, we used the mean of the two symmetric conditions, the mean of the two asymmetric conditions and the mean of the four unimanual conditions as input for the ANOVA.

The ANOVAs revealed a main effect for Symmetry on both RT $[F(2,18)=10.38, \quad P<0.01], \quad$ and MT $[F(2,18)=37.09, P<0.01]$. Post hoc analysis revealed that the unimanual trials (mean RT $=283 \mathrm{~ms}$, mean $\mathrm{MT}=790 \mathrm{~ms}$ ) 
Fig. 3 Overview of conditions (front view of CDs) used for the symmetry analysis and the endcomfort analysis. The top-left quadrant displays bimanual symmetric $(1,2)$ and asymmetric $(3,4)$ trials. The top-right quadrant displays unimanual control conditions for the symmetry analysis involving the left hand $(5,6)$ or the right hand $(7,8)$.

The conditions for the end comfort analysis are in the bottom half of the figure. At the bottomleft are the bimanual conditions with a comfortable end (overhand grip, 9) and with an uncomfortable end (underhand grip, 10), and at the bottom-right four unimanual control conditions involving the left hand $(11,12)$ or the right hand $(13,14)$

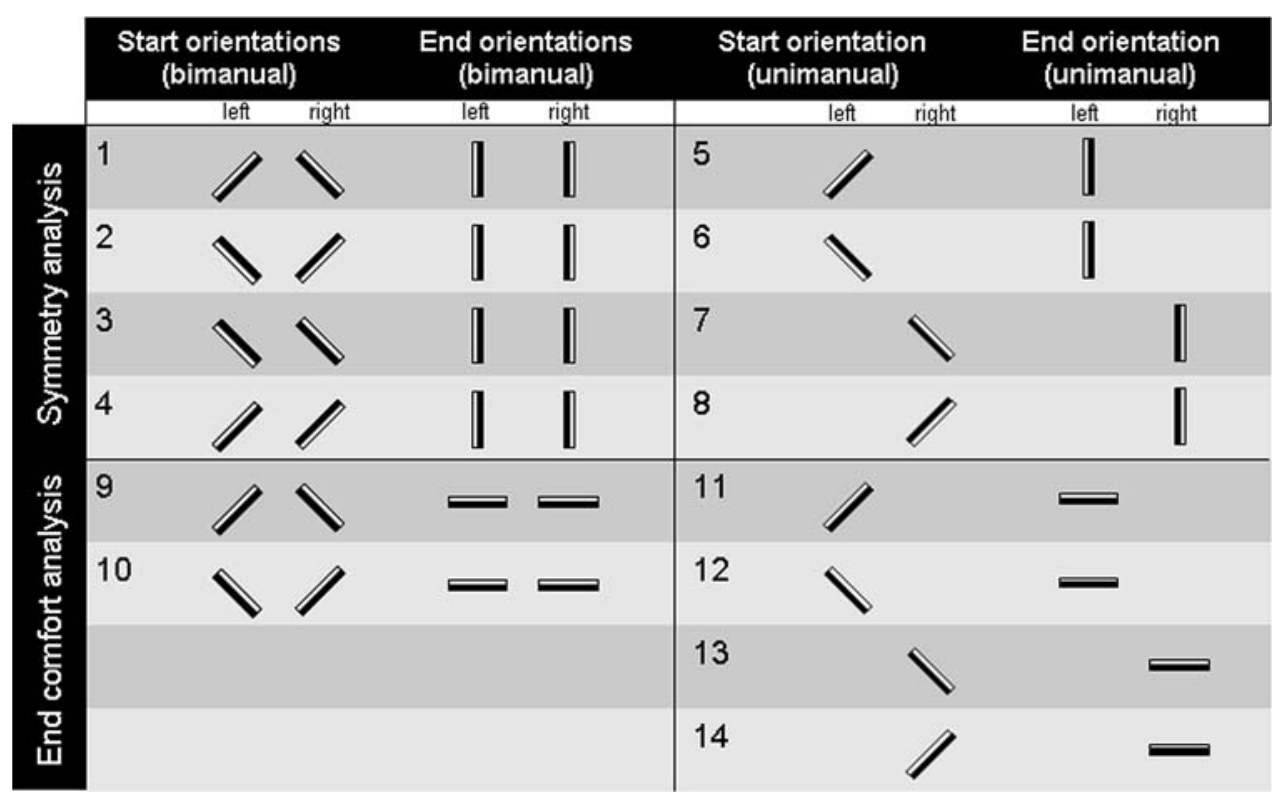

started earlier and had shorter movement times than both the symmetric trials (mean RT $=321 \mathrm{~ms}, t(9)=5.24$, $P<0.01$, mean MT $=877 \mathrm{~ms}, t(9)=7.15, P<0.01)$ and the asymmetric trials [mean $\mathrm{RT}=337 \mathrm{~ms}, t(9)=4.34$, $P<0.01$, mean MT $=908 \mathrm{~ms}, t(9)=10.67, P<0.01]$. Both RT and MT were not significantly different for symmetric and asymmetric movement trajectories $[t(9)=1.06$, n.s. and $t(9)=1.67$, n.s., respectively] .

\section{End comfort effects}

To identify the effects of end comfort on the kinematics, we performed two repeated measures ANOVAs (one for RT and one for MT) for which two bimanual conditions and four unimanual conditions were selected. The bimanual conditions both started non-parallel and ended horizontal. One of these conditions ended with an overhand (comfortable) grip, the other with an underhand (uncomfortable) grip (Fig. 3). Both conditions had symmetric movement trajectories, so that movement symmetry could not have confounded the results. In order to avoid interference with start posture comfort, vertical ending conditions were excluded from this analysis, as they required the less comfortable $135^{\circ}$ pronation start grip for an uncomfortable end. The four unimanual conditions were similar to the bimanual conditions, but then separated per hand (Fig. 3). The ANOVA design consisted of the following factors: Hand (two levels: left or right), Number of hands (two levels: 1 or 2) and End comfort (two levels: comfortable or uncomfortable).

The ANOVAs revealed main effects of Number of hands on both RT and MT, a main effect of End comfort on MT only, and no effects of Hand on RT or MT. As was also shown in the analyses of symmetry effects, the unimanual trials (mean RT $=285 \mathrm{~ms}$, mean MT $=841 \mathrm{~ms}$ ) started earlier $[F(1,9)=16.17, P<0.01]$ and had shorter movement times $[F(1,9)=57.28, P<0.01]$ than the bimanual trials (mean RT $=325 \mathrm{~ms}$, mean MT $=937 \mathrm{~ms}$ ). Although RTs were comparable for trials with comfortable and uncomfortable endings [mean RT $=308$ vs. $303 \mathrm{~ms}$, respectively, $F(1,9)<1$, n.s.], MT was significantly shorter in trials that ended comfortably [mean MT $=833$ vs. $944 \mathrm{~ms}$, respectively, $F(1,9)=19.67, P<0.01]$.

\section{Interlimb couplings}

In order to assess interlimb coupling strength we calculated Pearson's correlation coefficients ( $r$ values) of the rotations of the left and right wrists for the bimanual conditions involving symmetric or asymmetric movements and those involving comfortable or uncomfortable end grips (the same conditions used as for the symmetry and end comfort analyses). Fisher's $Z$ transformation was applied to the $r$ values before the analyses; average $Z$ values were retransformed to $r$ values for graphical display purposes.

Paired $t$ tests showed that interlimb coupling, as reflected by the correlation coefficient between the forearm rotations, was lower in asymmetric movements compared to symmetric movements [mean correlation $=0.78$ vs. 0.90 , respectively, $t(9)=2.46, P<0.05$, see Fig. 4], and higher in the trials that ended uncomfortably compared to those that ended comfortably [mean correlation $=0.98$ vs. 0.86, respectively; $t(9)=10.92, P<0.01$; see Fig. 4]. Moreover, the high correlation coefficients for symmetric and uncomfortable ending movements had, after the Fisher $Z$ transformation, a small range whereas the smaller correlation 
Fig. 4 Interlimb correlation coefficients for symmetric versus asymmetric movements (left) and for movements that end comfortable versus movements that end uncomfortable (right). Box plots show the median, interquartile range, outliers and extreme cases
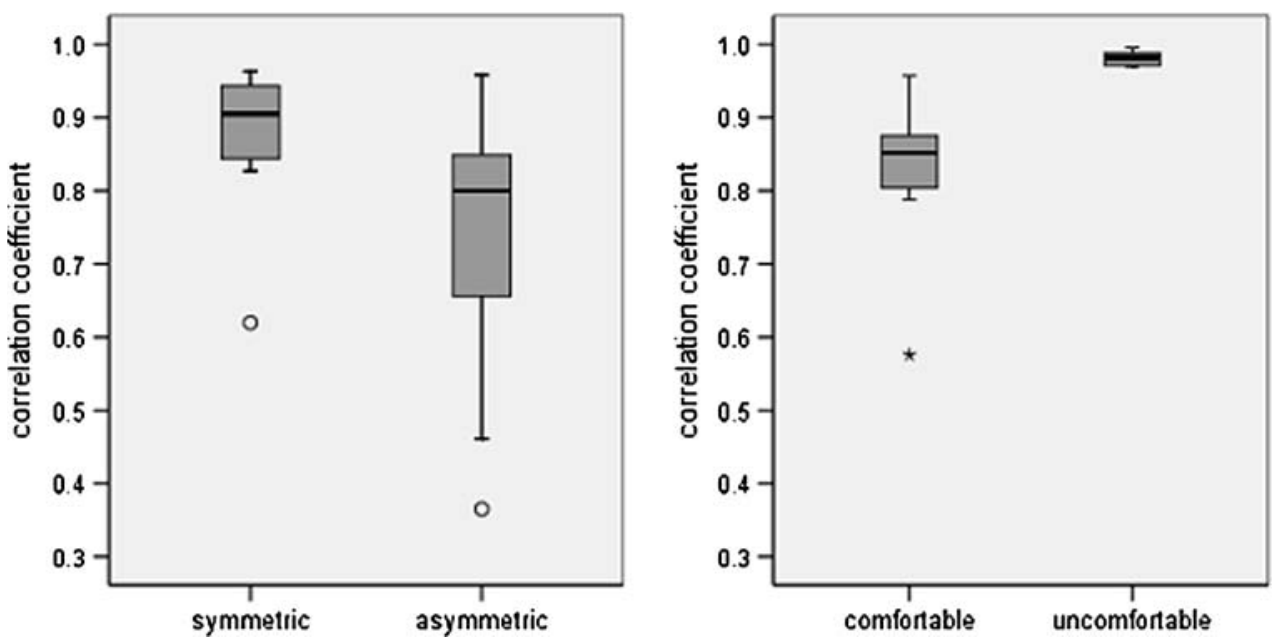

coefficients for the asymmetric and comfortable ending movements had a larger range (Fig. 4), which is another indication that the interlimb coupling was less strong in the latter conditions.

In addition, we scrutinized whether the correlation coefficients differed between the acceleration phase (before peak speed) and the deceleration phase (after peak speed) using a repeated measures ANOVA with a 2 (Time, acceleration vs. deceleration phase) $\times 2$ (End comfort, comfortable vs. uncomfortable) factor design. The factor End comfort again showed a significant effect on interlimb coupling $[F(1,9)=34.20, P<0.01]$. However, the factor Time was not significant $[F(1,9)=1.58$, n.s.]. The absence of both a main effect of Time and interaction between Time and End comfort $[F(1,9)=3.84$, n.s. $]$ indicates that the interlimb coupling was higher in trials that ended in a comfortable posture compared to trials that ended in an uncomfortable posture. This held for both the acceleration (mean correlation $=0.97$ vs. 0.86 , respectively) and the deceleration phase (mean correlation $=0.89$ vs. 0.82 , respectively).

\section{Discussion (1)}

In this first experiment, we studied the effect of end comfort and movement symmetry on the coordination of bimanual movement performance. As expected, and in line with previous studies (Swinnen et al. 1991, 1997; Carson 1995), interlimb coupling was lower when moving asymmetrically as compared to moving symmetrically. Interestingly, ending with both hands in an uncomfortable posture yielded a higher interlimb coupling than ending in a comfortable posture, both before and after peak velocity. These findings show that interlimb coordination is affected by the end posture during discrete bimanual tasks, even when this end posture is explicitly instructed via direct cueing.

In our second experiment we focus on the interaction between both constraints. In contrast to the first experiment, where start grip and end posture were explicitly instructed, participants in the second experiment were free to choose their start grips and the way in which they executed the movement. This set-up enabled us to study the interaction between posture planning and movement execution directly.

\section{Experiment 2}

The setup of the second experiment was comparable to the first experiment. However, participants could now freely choose their grip type with which they grasped the CDs before placing them in the $\mathrm{CD}$ boxes in a prescribed orientation. Unlike "Experiment 1", "Experiment 2" is not a reaction-time study. Instead, we analyzed the movement symmetry and end comfort of the hands for which we reported the direction of forearm rotation and the grip type that participants adopted.

Methods (2)

\section{Participants}

Ten right handed university students (two males and eight females, mean age $=20.6$ years, $\mathrm{SD}=1.9$ years) participated in the experiment. These students did not participate in the first experiment. They were all right-handers, as confirmed by a score of $\geq 60$ on the ten-item version of the Edinburgh handedness inventory (Oldfield 1971).

\section{Apparatus}

The experimental setup was similar to that used in the first experiment, with the exception that we now used four $\mathrm{CD}$ boxes (two upper boxes and two lower boxes, Fig. 5). In both the upper and the lower CD boxes the CDs could be 


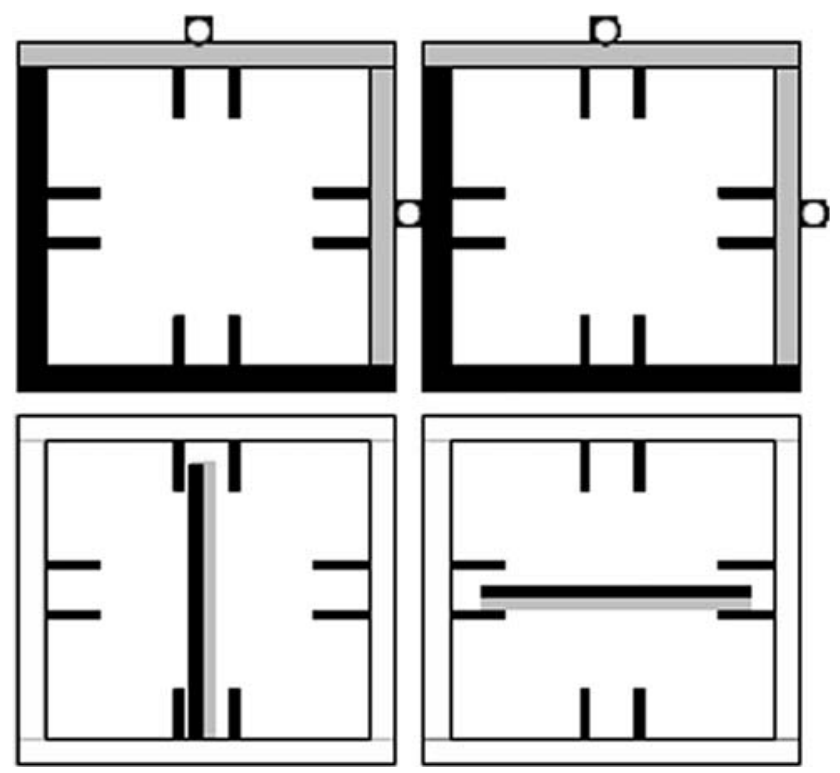

Fig. 5 Schematic drawing of the experimental setup. CDs have one black side and one green side (displayed gray) and are located in the lower boxes. The small circles on the top and right side of the upper boxes represents the LEDs. The upper boxes are covered with strips of green (displayed gray) and black paper

placed either horizontally or vertically. The two upper boxes had green LEDs on the upper and right sides, which indicated the required end orientation of the CDs. In addition, and as a further cue, the borders of the upper boxes were covered with strips of green (on the upper and right sides) and black (on the left and lower sides) paper. The CDs also had a green and a black side which enabled us to indicate either a $0^{\circ}$ orientation or a $180^{\circ}$ orientation (and either $90^{\circ}$ pronation or $90^{\circ}$ supination) by the instruction that the $\mathrm{CD}$ had to be placed with the green side facing the green LED.

\section{Task}

Participants started with their hands on the table, in front of the $\mathrm{CD}$ boxes and they had to pick up the two CDs simultaneously from the lower boxes and place them in the upper boxes. Using this setup, participants were left free with respect to the type of grip with which they grasped the CDs. The CDs had to be placed with their green side towards the green LEDs. Thus, when the LED on the upper side turned on, the CD had to be placed horizontally with the green side up, and when the LED on the right side turned on, the CD had to be placed vertically with the green side to the right.

\section{Experimental design}

The experiment consisted of 24 conditions in which we manipulated the start and end orientation of the CDs, the start and end orientation congruency and the required rotation. The start and end orientation of each CD could be either horizontal or vertical. Start and end orientation congruency could be either congruent (i.e., both CDs horizontal or both CDs vertical) or incongruent (i.e. one CD horizontal and the other $\mathrm{CD}$ vertical). The required rotation could be $0^{\circ}, 90^{\circ}$ supination, $90^{\circ}$ pronation or $180^{\circ}$. In theory, participants could also make a rotation of $270^{\circ}$ in the opposite direction, but we assumed that they would choose the shortest angle of rotation.

Conditions were such that one CD always had to be rotated $180^{\circ}$ to place it horizontally or vertically. The required rotation for the other $\mathrm{CD}$ was $0^{\circ}, 90^{\circ}$ pronation or $90^{\circ}$ supination. These manipulations resulted in 2 possibilities for the $180^{\circ}$ rotating arm ( 2 orientations: horizontal or vertical) and 6 possibilities for the other arm (3 rotations $\times 2$ orientations). As the rotations could be performed with either hand, this resulted in a total of $2 \times 2 \times 6=24$ conditions. Conditions of interest were those that had a conflict between moving symmetrically and ending comfortably. There were four conditions with such a conflict, an example of which is given in Fig. 6.

Participants performed 120 trials that were administered in five blocks of the 24 conditions in a randomized order. Trials within a block were repeated at the end of that block in case the participant ended the movement in the wrong orientation. Before the start of the experimental trials, participants performed 12 practice trials to check whether the task was understood correctly, and to familiarize themselves with the task. The total duration of the experiment was about $1 \mathrm{~h}$.

\section{Comfort ratings}

Similar to the first experiment, participants rated comfort of the start and end postures before and after the experimental trials. Again, the mean score of these two measurements was used for further analysis. As the start and end grips were not prescribed, all postures that were reasonably possible (biomechanically) were rated for their comfort. These included the four postures in Fig. 2 denoted as end postures and also one additional vertical, supinated grip with the thumb down. Together, this resulted in five grips ( 2 horizontal and 3 vertical) $\times 2$ hands $\times 2$ orientations (start and end orientation $)=20$ comfort ratings.

\section{Data analysis}

For each experimental trial we registered the rotation of the hand (pronation or supination) and the side of the thumb on the $\mathrm{CD}$ (on the green or on the black side) while grasping the $\mathrm{CD}$. For this purpose, experimental trials were videotaped. The side of the thumb on the $\mathrm{CD}$ was used to categorize the 


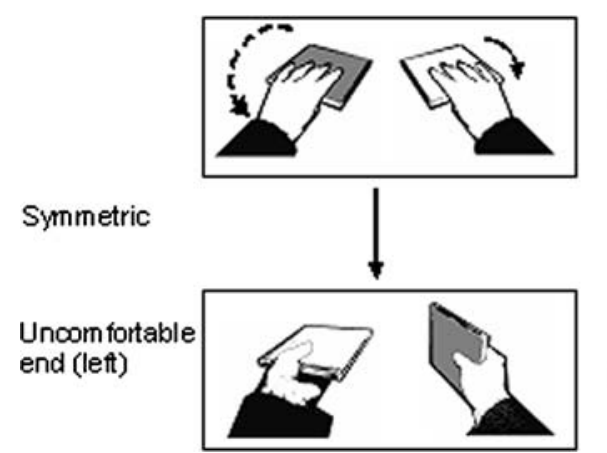

Fig. 6 An experimental condition containing a conflict between moving the hands symmetrically and ending comfortable. The start orientation was for both hands horizontal. The end orientation for the left $\mathrm{CD}$ was horizontal, requiring a rotation of $180^{\circ}$; the end orientation for the right hand was vertical, requiring a rotation of $90^{\circ}$ supination. The

adopted start and end postures into one of five possible options: a horizontal overhand posture, a horizontal underhand posture, a vertical posture with the thumb pointing up and two vertical postures with the thumb pointing down (one in pronation and one in supination). The comfort ratings were used to categorize the postures as either comfortable or uncomfortable. Based on the rotation of both hands, movements were scored as symmetrically (both hands pronation or both hands supination) or asymmetrically (one hand pronation and the other hand supination).

Depending on the research question and type of dependent variable under investigation, we applied repeated-measures ANOVAs and paired $T$ tests, which will be described separately in the relevant paragraphs of the results section.

\section{Results (2)}

\section{Comfort ratings}

Participants gave ratings for five different start postures and five different end postures (Table 1). For this experiment, however, we only analyzed the end postures. We included all conditions in the analyses and the start orientations were balanced across conditions so that start posture comfort could not confound the results. As the ratings given before the experiment did not differ from those given after the experiment, we used the mean ratings for end comfort in a repeated measures ANOVA. The design of this ANOVA consisted of two within-subject factors; Hand (two levels: left or right), and End posture (five levels: horizontal overhand, horizontal underhand, vertical with thumb up, vertical pronated with thumb down and vertical supinated with thumb down). The ANOVA revealed a significant effect of End posture $[F(4,6)=78.8, P<0.001]$, but not for Hand $[F(1,9)=3.64, P=0.089]$. Post hoc pair wise comparisons showed that for the horizontal postures, the overhand grip was rated more comfortable than the underhand grip

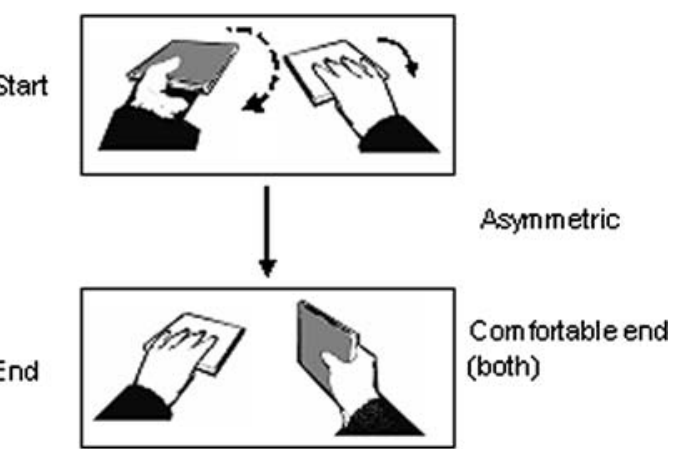

rotation direction of the left hand could be supination (depicted on the left), resulting in a symmetrical movement trajectory, but an uncomfortable end posture. Alternatively, the rotation direction of the left hand could be pronation (depicted on the right), resulting in an asymmetric movement trajectory, but a comfortable end posture

Table 1 Mean (and standard deviation) of the comfort ratings assessed by means of a 5-point scale for start and end postures studied in Experiment 2

\begin{tabular}{lllll}
\hline Hand & Orientation & Posture & Start & End \\
\hline Left & Horizontal & Overhand & $4.20(0.95)$ & $4.65(0.67)$ \\
& & Underhand & $4.30(1.03)$ & $3.85(1.18)$ \\
& \multirow{2}{*}{ Vertical } & Thumb up & $4.75(0.55)$ & $4.85(0.37)$ \\
& & Thumb down & $2.10(1.21)$ & $2.55(1.28)$ \\
& & Thumb down & $1.40(0.68)$ & $1.35(0.59)$ \\
Right & Horizontal & Overhand & $4.70(0.66)$ & $4.95(0.22)$ \\
& & Underhand & $4.05(1.32)$ & $3.25(1.21)$ \\
& \multirow{4}{*}{ Vertical } & Thumb up & $4.90(0.31)$ & $4.85(0.49)$ \\
& & Thumb down & $2.05(1.10)$ & $2.30(1.03)$ \\
& & Thumb down & $1.20(0.41)$ & $1.30(0.57)$ \\
\hline
\end{tabular}

$[t(9)=5.175, P=0.001]$. For the vertical start orientations, a grip type with the thumb pointing up was rated significantly more comfortable than both a pronated grip with the thumb pointing down $[t(9)=8.195, P<0.001]$ and a supinated grip with the thumb pointing down $[t(9)=23.321$, $P<0.001]$.

In sum, for the end postures a horizontal overhand grip and a vertical grip with the thumb pointing up were considered as comfortable. Conversely, a horizontal underhand grip and both vertical grips with the thumb pointing down were considered as uncomfortable.

\section{Symmetry effects}

Frequencies of moving symmetrically were analyzed in conditions in which one hand rotated $180^{\circ}$ and the other hand rotated $90^{\circ}$. Trials in which no rotation was required for one hand (eight conditions) or in which participants rotated $270^{\circ}$ instead of $90^{\circ}$ (5.3\% of these trials) were discarded from the analysis. 
The hands moved symmetrically in $46.2 \%$ ( $\mathrm{SD}=6.1 \%$ ). A repeated measures ANOVA with factors Hand (hand that rotated $180^{\circ}$ : left or right) and End orientation (end orientation of the $\mathrm{CD}$ that had to be rotated $180^{\circ}$ : horizontal or vertical) revealed no difference between trials in which the left hand rotated $180^{\circ}$ and those in which the right hand rotated $180^{\circ}$. However, the number of symmetric movements was higher when the end orientation of the $180^{\circ}$ rotating CD was horizontal (mean symmetric movements $=49.7 \%$, $\mathrm{SD}=6.0 \%$ ) compared to vertical (mean symmetric movements $=42.7 \%, \quad \mathrm{SD}=6.87 \% ; \quad \mathrm{F}(1,9)=6.054$, $\mathrm{p}=0.036$, Fig. 7).

\section{End comfort effects}

For the analysis of end comfort effects, we included all conditions. The right hand ended comfortably in $82.0 \%$ $(\mathrm{SD}=20.2 \%)$ of all trials, compared to only $49.8 \%$ $(\mathrm{SD}=9.8 \%)$ for the left hand. This rather large variability for the right hand suggests that the end-state comfort effect is not consistent across task conditions. Therefore, we analyzed the effects of end orientation and rotation angle on the end postures of both hands using a repeated measures ANOVA including the factors Hand (left or right), End orientation (horizontal or vertical) and Rotation angle $\left(0^{\circ}, 90^{\circ}\right.$ or $180^{\circ}$ ). This ANOVA revealed that participants ended more frequently in a comfortable posture when the end orientation was vertical (mean comfortable ends $=80.8 \%$, $\mathrm{SD}=11.3 \%$ ) compared to horizontal [mean comfortable ends $=61.9 \%, \quad \mathrm{SD}=15.7 \% ; \quad F(1,9)=5.842, \quad P=0.039$,

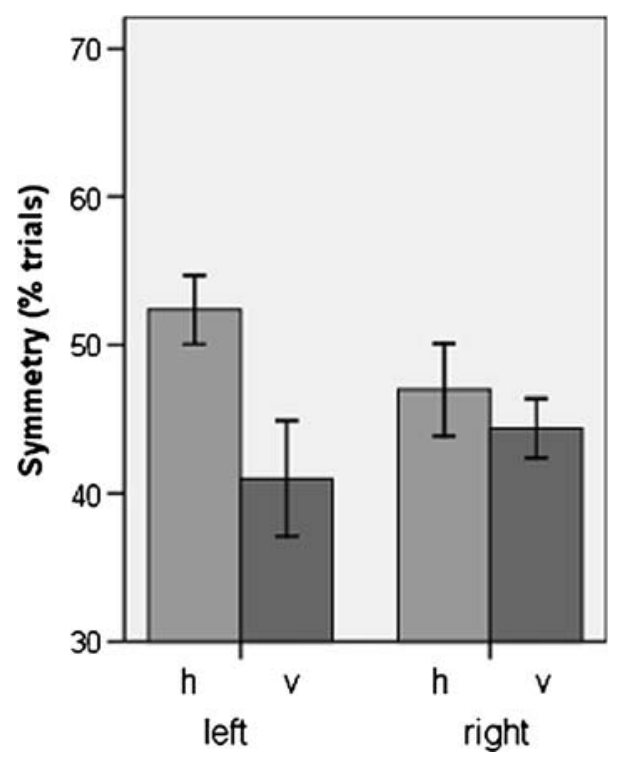

Fig. 7 Percentages of trials in which the hands moved symmetrically. The trials were separated for those in which the left hand rotated $180^{\circ}$ and those in which the right hand rotated $180^{\circ}$, as well as the end orientation of this hand. $h=$ horizontal end orientation; $v=$ vertical end orientation. Error bars reflect Standard Errors
Fig. 8]. In addition, there was an effect of rotation angle $[F(2,18)=8.204, P=0.011]$ : the larger the rotation angle, the smaller the number of comfortable endings.

As the percentage of comfortable end postures for the left hand was surprisingly low, we examined this matter in more detail. Therefore, we scrutinized the performance of the left hand in the bimanual trials. It appeared that for trials that had similar start orientations (i.e. both CDs horizontal or both CDs vertical at the start), the participants adopted predominantly the same start postures for both hands in $60.1 \%$ when the left hand ended uncomfortably. In trials in which the left hand ended comfortably the participants adopted a symmetrical start posture in only $29.7 \%$. However, when the end orientations of the CDs were similar, the participants adopted the same end postures for both hands in only $15.5 \%$ when the left hand ended uncomfortably. In trials in which the left hand ended comfortably the participants adopted a symmetrical end posture in $79.9 \%$. Thus, for the left hand, participants predominantly preferred a similar start posture, but not a similar end posture to the right hand, which finally resulted in an uncomfortable end posture.

\section{Discussion (2)}

In the second experiment, we investigated the preference of participants either to end comfortably or move symmetrically in the bimanual CD-displacement task. As expected, and in line with previous studies on bimanual object manipulations (Fischman et al. 2003; Weigelt et al. 2006) partici-

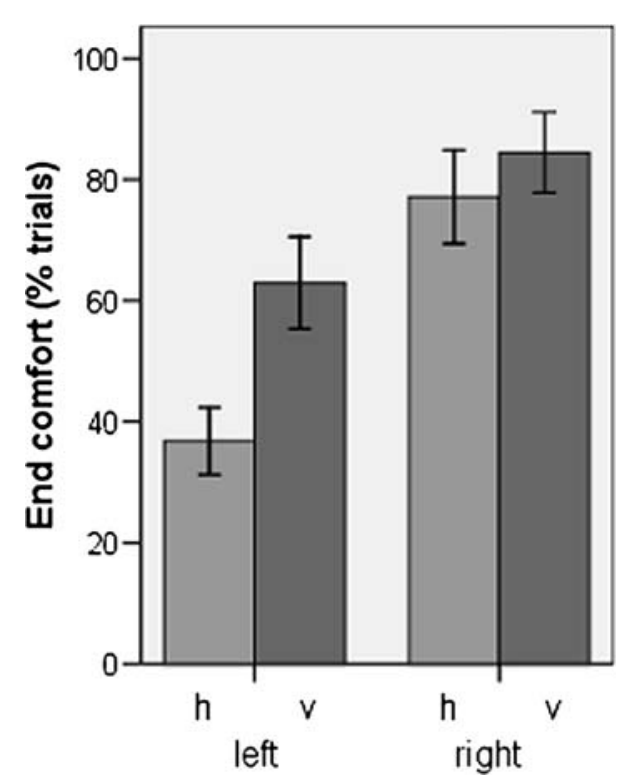

Fig. 8 Percentages of trials in which the hands ended in a comfortable posture. The trials were separated for the left and the right hand, and for a horizontal $(h)$ and a vertical $(v)$ end orientation. Error bars reflect Standard Errors 
pants chose to end comfortably, regardless of whether the comfortable end posture was reached by symmetric or asymmetric rotations of the forearms. Interestingly, the end-state comfort effect predominantly affected the right hand. These findings indicate that planning of comfortable goal states is limited in complex bimanual object manipulation tasks, but at the same time is a more powerful constraint than movement symmetry.

\section{General discussion}

The primary aim of the two experiments was to examine the relative importance of planning and execution constraints in discrete bimanual object manipulation. The main finding in "Experiment 1" was the presence of an effect of a prescribed and cued end posture on interlimb coupling. The second experiment showed that planning constraints dominated execution constraints. This was reflected by the preference to end comfortably and an absence of a preference to move both hands in symmetry. Moreover, planning for comfortable end postures was only found to the right hand. We will elaborate on these new findings below. Apart from these new findings we replicated some well known phenomena from the literature. That is, reaction times and movement times were longer in bimanual trials compared to unimanual trials (e.g., Kelso et al. 1979; Jackson et al. 2002; Mason and Bryden 2007), and interlimb coupling was higher when moving in symmetry than in asymmetry (e.g., Swinnen et al. 1991; Carson 1995; Semjen et al. 1995).

\section{Symmetry effects}

The results of the second experiment revealed that in less than $50 \%$ of the trials participants chose to move symmetrically, i.e. almost at chance level. This finding is in strong contrast to that in cyclical tasks where symmetry of moving dominates. Particularly when high-speed requirements are imposed on the participant, there is a ubiquitous tendency for interlimb synchronization, as reflected by the occurrence of spontaneous transitions from the asymmetric to the symmetric coordination mode and the high degree of stability and accuracy in this latter mode (Byblow et al. 1994; Swinnen et al. 1997; Carson et al. 2000). The present results also argue against the parameter-specification model of Heuer (1993). In our second experiment, the participants were well able to specify different movement parameters for each hand (e.g., rotation angle, rotation direction) in order to end comfortably, without much interference effects (i.e. the tendency to move symmetrically). The present results rather suggest that the role of symmetry depends on the nature of the task. In discrete, goal-directed movements, which require that the goal of the movement is planned in advance, the symmetry constraint may play a subordinate role, or may even be absent, in contrast to cyclical movements. Moreover, Kunde and Weigelt (2005) showed that in a discrete task, symmetry effects only became apparent when the movements had no other goal than carrying out the movements itself, instead of moving towards a perceptual goal. Weigelt et al. (2006) also found a subordinate role for means-related influences at the expense of endsrelated influences on action planning in discrete, goaldirected, movements. However, in their study, meansrelated was attributed to the symmetry of the initial handgrips instead of the symmetry of moving while manipulating the object. The present study extends the findings of Weigelt et al. (2006) by showing that the selection of handgrips is not driven by the preference to move in symmetry.

In our first experiment, the absence of RT and MT differences between the symmetric and asymmetric movements also implies that movement symmetry did not affect performance (see also Kunde and Weigelt 2005). However, our first experiment did show a strong interlimb coupling in symmetric conditions and a weak coupling in asymmetric conditions, which was shown previously in cyclical movements (Carson 1995; Swinnen et al. 1997) and in discrete movements. With respect to the latter, Kunde and Weigelt (2005) demonstrated synchronicity in bimanual object depositing in symmetric compared to asymmetric trials. The interlimb coupling in the present study was calculated over the interval from reaction time until the end of the movement, thus including object depositing. A higher coupling when moving symmetrically, may be explained by the involvement of homologous muscle pairs that may have a centrally specified pattern of excitation, in contrast to the non-homologous muscle pairs active during asymmetric movements (Carson 1995). Nevertheless, the potential influence of simultaneous activation of homologous muscle pairs on the coordination of discrete bimanual objectmanipulation is limited as only the interlimb coupling was affected and not the RT, MT, or the choice for a grip type.

\section{End comfort effects}

In contrast to moving symmetrically, participants did choose to end the movements in the second experiment with a comfortable posture, at least for their right hand. Surprisingly, the left hand ended in a comfortable end posture only in half of the trials. Participants preferred similar start postures instead, which often resulted in an uncomfortable end posture. These findings are in contrast with those of Weigelt et al. (2006), who demonstrated the end-state comfort effect for both hands in discrete bimanual object manipulations. However, the task and conditions that they used were less complex, i.e., the objects always had the 
same end orientation, i.e. congruent, whereas the objects in our experiment also had to be placed in different end orientations. We therefore suggest that the planning of a comfortable end posture for both hands depends on the complexity of the task. Increase in complexity of the task, and its concomitant larger cognitive load leads to a 'breakdown' of anticipatory planning of both hands, such that only the end-state comfort of the right hand is anticipated, but not of the left hand. In addition, we showed that the end-state comfort effect was reduced with larger rotation angles. This further hints to the suggestion that advance goal state planning relates to the complexity of the task, which increases with larger rotation angles.

The effects of the end-comfort constraint on the kinematics of discrete bimanual object manipulations have, to our knowledge, not been demonstrated before. Although a major role is ascribed to the influence of end comfort on the selection of macroscopic handgrips for object manipulations, we showed that this constraint also affected the kinematics of movement execution. The interlimb coupling was stronger in uncomfortable ending trials than in comfortable ending trials. This difference might have a biomechanical origin. In an awkward arm-hand position, that is, in trials with an uncomfortable end posture, the hand has less freedom to move compared to a comfortable position when the hand is in the middle of the range of motion (Rossetti et al. 1994). As a consequence, coupling may be stronger. If this hypothesis holds, then the difference in interlimb coupling between comfortable and uncomfortable ending trials should be particularly large at the end of the movement, thus after peak velocity. In contrast, we found that interlimb coupling was higher in trials ending uncomfortably both before and after peak velocity, making a biomechanical explanation of these differences unlikely. A more reasonable explanation for this difference in coupling strength is that comfortable ending trials are more experienced and that practice results in a freeing of the degrees of freedom of the action system that underlies interlimb coordination (Temprado et al. 1997).

\section{Hemispheric specialization}

Recent research has shown that the hemispheres have a specialized role in the control of motor actions (Gonzalez et al. 2006; Serrien et al. 2006). However, these studies focused on the execution of hand movements, whereas we showed a difference in planning of end comfort between the hands, which precedes the execution. Haaland et al. (2004) did show left hemisphere dominance for planning of complex movement sequences, but not only for the right hand, also for the left hand. Sainburg and Schaefer (2004) examined interlimb differences regarding both planning and execution. They distinguished two features of movement control that contributed in reaching a certain peak velocity: (1) Pulse-height control, which is the adjustment of the amplitude of the initial acceleration impulse as a result of preplanning and (2) Pulse-width control, which is the adjustment of the duration of the initial acceleration impulse using sensory feedback. In right-handed individuals, they showed that the dominant hemisphere/limb system (left hemisphere, right hand) relied more on pulse-height control, whereas the non-dominant hemisphere/limb system (right hemisphere, left hand) relied more on pulse-width control. The propensity of the dominant hemisphere/limb system to use preplanning (feedforward) instead of feedback mechanisms to reach a certain peak velocity is in line with the present finding of anticipatory planning of end comfort for the right hand. However, it yet has to be established if the processes involved in preplanning of the initial acceleration impulse are connected or even similar to the processes involved in planning of such a macroscopic variable as grip type. Furthermore, on the basis of the present findings it cannot be verified whether the difference in endstate comfort effect between both hands is a result of hemispheric specialization or simply handedness, as all participants in the present study were right-handed. An experiment with left-handers could resolve that issue.

In conclusion, we showed that the coordination between the hands in bimanual object manipulations was subordinate to the planning of the movement execution. However, this planning constraint was only present for the right hand, which may be due to differences in hemispheric specializations regarding motor planning.

Open Access This article is distributed under the terms of the Creative Commons Attribution Noncommercial License which permits any noncommercial use, distribution, and reproduction in any medium, provided the original author(s) and source are credited.

\section{References}

Bingham GP, Hughes K, Mon-Williams M (2008) The coordination patterns observed when two hands reach-to-grasp separate objects. Exp Brain Res 184:283-293

Byblow WD, Carson RG, Goodman D (1994) Expressions of asymmetries and anchoring in bimanual coordination. Hum Mov Sci $13: 3-28$

Carson RG (1995) The dynamics of isometric bimanual coordination. Exp Brain Res 105:465-476

Carson RG, Riek S, Smethurst CJ, Parraga JFL, Byblow WD (2000) Neuromuscular-skeletal constraints upon the dynamics of unimanual and bimanual coordination. Exp Brain Res 131:196-214

Diedrichsen J, Hazeltine E, Kennerley S, Ivry RB (2001) Moving to directly cued locations abolishes spatial interference during bimanual actions. Psychol Sci 12:493-498

Fischman MG, Stodden DF, Lehman DM (2003) The end-state comfort effect in bimanual grip selection. Res Q Exerc Sport 74:17-24 
Gonzalez CLR, Ganel T, Goodale MA (2006) Hemispheric specialization for the visual control of action is independent of handedness. J Neurophysiol 95:3496-3501

Haaland KY, Elsinger CL, Mayer AR, Durgerian S, Rao SM (2004) Motor sequence complexity and performing hand produce differential patterns of hemispheric lateralization. J Cogn Neurosci 16:621-636

Heuer H (1993) Structural constraints on bimanual movements. Psychol Res Psychol Forsch 55:83-98

Hughes CML, Franz EA (2008) Goal-related planning constraints in bimanual grasping and placing of objects. Exp Brain Res 188:541-550

Jackson GM, German K, Peacock K (2002) Functional coupling between the limbs during bimanual reach-to-grasp movements. Hum Mov Sci 21:317-333

Johnson-Frey SH, McCarty ME, Keen R (2004) Reaching beyond spatial perception: effects of intended future actions on visually guided prehension. Vis Cogn 11:371-399

Kelso JAS, Southard DL, Goodman D (1979) Coordination of 2-handed movements. J Exp Psychol Hum Percept Perform 5:229-238

Kunde W, Weigelt M (2005) Goal congruency in bimanual object manipulation. J Exp Psychol Hum Percept Perform 31:145-156

Li Y, Levin O, Carson RG, Swinnen SP (2004) Bimanual coordination: constraints imposed by the relative timing of homologous muscle activation. Exp Brain Res 156:27-38

Marteniuk RG, Mackenzie CL, Jeannerod M, Athenes S, Dugas C (1987) Constraints on human arm movement trajectories. Can J Psychol 41:365-378

Mason AH, Bryden PJ (2007) Coordination and concurrency in bimanual rotation tasks when moving away from and toward the body. Exp Brain Res 183:541-556

Oldfield RC (1971) Assessment and analysis of handedness-Edinburgh inventory. Neuropsychologia 9:97-113

Rosenbaum DA, Barnes HJ, Vaughan J, Jorgensen MJ (1992) Time course of movement planning-selection of handgrips for object manipulation. J Exp Psychol Learn Mem Cogn 18:1058-1073

Rosenbaum DA, Meulenbroek RJ, Vaughan J, Jansen C (2001) Posture-based motion planning: applications to grasping. Psychol Rev 108:709-734
Rossetti Y, Meckler C, Prablanc C (1994) Is there an optimal arm posture-deterioration of finger localization precision and comfort sensation in extreme arm-joint postures. Exp Brain Res 99:131-136

Sainburg RL, Schaefer SY (2004) Interlimb differences in control of movement extent. J Neurophysiol 92:1374-1383

Semjen A, Summers JJ, Cattaert D (1995) Hand coordination in bimanual circle drawing. J Exp Psychol Hum Percept Perform 21:1139-1157

Serrien DJ, Ivry RB, Swinnen SP (2006) Dynamics of hemispheric specialization and integration in the context of motor control. Nat Rev Neurosci 7:160-167

Spijkers W, Heuer H (1995) Structural constraints on the performance of symmetrical bimanual movements with different amplitudes. Q J Exp Psychol Sect A Hum Exp Psychol 48:716-740

Spijkers W, Heuer H, Kleinsorge T, van der Loo H (1997) Preparation of bimanual movements with same and different amplitudes: specification interference as revealed by reaction time. Acta Psychol 96:207-227

Steenbergen B, Marteniuk RG, Kalbfleisch LE (1995) Achieving coordination in prehension: joint freezing and postural contributions. J Mot Behav 27:333-348

Swinnen SP, Jardin K, Meulenbroek R, Dounskaia N, HofkensVan Den Brandt M (1997) Egocentric and allocentric constraints in the expression of patterns of interlimb coordination. J Cogn Neurosci 9:348-377

Swinnen SP, Jardin K, Verschueren S, Meulenbroek R, Franz L, Dounskaia N, Walter CB (1998) Exploring interlimb constraints during bimanual graphic performance: effects of muscle grouping and direction. Behav Brain Res 90:79-87

Swinnen SP, Young DE, Walter CB, Serrien DJ (1991) Control of asymmetrical bimanual movements. Exp Brain Res 85:163-173

Temprado J, Della-Grasta M, Farrell M, Laurent M (1997) A noviceexpert comparison of (intra-limb) coordination subserving the volleyball serve. Hum Mov Sci 16:653-676

Weigelt M, Kunde W, Prinz W (2006) End-state comfort in bimanual object manipulation. Exp Psychol 53:143-148

Weigelt M, Rieger M, Mechsner F, Prinz W (2007) Target-related coupling in bimanual reaching movements. Psychol Res Psychol Forsch 71:438-447 